\title{
How urinary stone emergencies changed in the time of COVID-19?
}

\author{
Simone Flammia ${ }^{1}$. Stefano Salciccia ${ }^{1,2}$ (D) Antonio Tufano ${ }^{1} \cdot$ Gian Maria Busetto ${ }^{1}$. Gian Piero Ricciuti ${ }^{1}$. \\ Alessandro Sciarra ${ }^{1}$
}

Received: 27 April 2020 / Accepted: 15 May 2020 / Published online: 28 May 2020

(c) Springer-Verlag GmbH Germany, part of Springer Nature 2020

The pandemic acute respiratory syndrome coronavirus 2 (SARS-CoV-2), named COVID-19 [1], is generating a severe health emergency all over the world but particularly in some countries. The healthcare challenge is to provide assistance to the increasing number of infected patients, to contain ways of transmission and at the same time to treat all the non-deferrable medical conditions that continue to affect the population. Moreover, the most relevant problems are in the management of all first aid accesses and emergencies other than COVID-19. In this brief communication, we report our experience on the diagnostic and therapeutic procedures requested and performed for urinary stone emergencies during a 6-week period activity in a hospital partially converted to COVID-19 care and in the highest national level of COVID-19 infection (March-April 2020), with the management performed in the same hospital in a no-COVID-19 period (March-April 2019) 1 year ago. In particular, we analyzed differences between these two timerelated populations in terms of patients and urinary stone characteristics, symptoms and complications at presentation, diagnostic and therapeutic approaches, time of hospitalization. ANOVA analysis and Chi square test were used to quantify the differences between the two time periods. Some relevant data are obtained (Table 1): (1) independent to COVID-19 infection, in our hospital, the number of first aid accesses for urinary stone emergencies did not significantly change (44 cases in the no COVID-19 period versus 36 cases in the COVID-19 period) (2) patients presenting during COVID-19 time showed significantly higher serum levels of creatinine $(p=0.026)$ when compared to a noCOVID-19 period. These data may suggest a delay in terms of patient presentation to the hospital, related to the pandemic. (3) However, no significant differences were detected in terms of complication rates, urinary stone diameter or grade of hydronephrosis due to COVID-19 pandemic. (4) Stone position significantly changed with a higher rate of lumbar ureter and lower of juxta-vesical site during COVID19 period $(p=0.036)$. The reduction of first aid access for juxta-vesical site stones may be interpreted through a higher rate of its management using medical therapies at home. (5) Diagnostic and therapeutic approach to urinary stone emergencies did not significantly change compared to the non-pandemic period. In particular, the use of nephrostomy or ureteral stent for the first aid did not significantly change. Across a 6-week period during the peak of the COVID-19 pandemic, urinary stone emergencies continued to be managed with few significant variations in a geographical area at medium density for COVID-19 and in a hospital partially converted in first aid cares. An effective reorganization of health care facilities in hospitals can consent to do not reduce cares and resources for patients with other urgent and emergent conditions such as urinary stones [2-3]. The findings presented here suggest that urinary stone emergencies are mainly severe, their care need to be continued and they were not significantly influenced by this pandemic.

Stefano Salciccia

stefano.salciccia@uniroma1.it

1 Department of Urology, University Sapienza, Rome, Italy

2 Department of Urological Sciences, University Sapienza, Viale Policlinico 155, 0016 Rome, Italy 
Table 1 Patient characteristics: percentage of cases (\%); mean $\pm \mathrm{SD}$ and range

\begin{tabular}{|c|c|c|c|}
\hline Variable & 1st March-15th April 2019 & 1st March-15th April 2020 & $p$ value \\
\hline Age (years) & $56.55 \pm 15.98(40-74)$ & $54.25 \pm 18.54(35-74)$ & 0.776 \\
\hline Weight (kg) & $69.18 \pm 9.53(60.0-84.0)$ & $89.00 \pm 15.26(72.0-97.0)$ & 0.003 \\
\hline BMI $\left(\mathrm{kg} / \mathrm{m}^{2}\right)$ & $25.11 \pm 3.85(20-29)$ & $26.87 \pm 4.07(20-32)$ & 0.349 \\
\hline Charlson Comorbidity Index & & & 0.658 \\
\hline 0 & $36.4 \%$ & $50.0 \%$ & \\
\hline 1 & $18.2 \%$ & $0.0 \%$ & \\
\hline$\geq 2$ & $45.5 \%$ & $50.0 \%$ & \\
\hline sCR at ED entry (mg/dl) & $1.20 \pm 0.54(0.80-1.70)$ & $2.87 \pm 1.25(1.25-10.93)$ & 0.026 \\
\hline WBC at ED entry $(1000 \times \mathrm{UI} / \mathrm{ml})$ & $13.13 \pm 5.19(7.0-19.0)$ & $8.71 \pm 3.49(5.0-12.0)$ & 0.052 \\
\hline Previous history of urinary stones & & & 0.370 \\
\hline Negative & $63.6 \%$ & $37.5 \%$ & \\
\hline Positive & $36.4 \%$ & $62.5 \%$ & \\
\hline Renal colic pain as main symptom & & & 0.959 \\
\hline Absent & $27.3 \%$ & $25.0 \%$ & \\
\hline Present & $72.7 \%$ & $75.0 \%$ & \\
\hline Complicated UTI at ED entry & & 7 & 0.659 \\
\hline Absent & $63.6 \%$ & $75.0 \%$ & \\
\hline Present & $36.4 \%$ & $25.0 \%$ & \\
\hline Imaging test at ED & & & 0.945 \\
\hline US and/or X-ray & $45.5 \%$ & $37.5 \%$ & \\
\hline CT scan only & $18.2 \%$ & $12.5 \%$ & \\
\hline Both & $36.4 \%$ & $50.0 \%$ & \\
\hline Hydronefrosis at ED entry & & & 0.955 \\
\hline Absent & $18.2 \%$ & $12.5 \%$ & \\
\hline Present & $81.8 \%$ & $87.5 \%$ & \\
\hline Side of hydronefrosis & & & 0.247 \\
\hline Right & $45.5 \%$ & $50.0 \%$ & \\
\hline Left & $45.5 \%$ & $12.5 \%$ & \\
\hline Bilateral & $9.0 \%$ & $37.5 \%$ & \\
\hline Grade of hydronephrosis & & & 0.633 \\
\hline $0-1$ & $45.5 \%$ & $25.0 \%$ & \\
\hline $2-3$ & $54.5 \%$ & $75.0 \%$ & \\
\hline Number of stones & & & 0.965 \\
\hline 1 & $63.6 \%$ & $62.5 \%$ & \\
\hline $2-5$ & $27.3 \%$ & $25.0 \%$ & \\
\hline$>5$ & $9.1 \%$ & $12.5 \%$ & \\
\hline Stone position & & & 0.036 \\
\hline Renal (ampulla, and/or calices) & $36.4 \%$ & $25.0 \%$ & \\
\hline Lumbar ureter & $0.0 \%$ & $50.0 \%$ & \\
\hline Juxta-vesical ureter & $54.5 \%$ & $12.5 \%$ & \\
\hline Bladder & $9.1 \%$ & $12.5 \%$ & \\
\hline Maximum diameter stone (mm) & $10.0 \pm 4.3(6-16)$ & $8.0 \pm 2.8(6-13)$ & 0.442 \\
\hline Nephrostomy at ED & & & 0.181 \\
\hline None & $8(72.7 \%)$ & $37.5 \%$ & \\
\hline Yes & $3(27.3 \%)$ & $62.5 \%$ & \\
\hline Stone treatment & & & 0.578 \\
\hline Medical therapy only & $45.5 \%$ & $37.5 \%$ & \\
\hline RIRS & $9.1 \%$ & $0.0 \%$ & \\
\hline ULT & $18.2 \%$ & $50.0 \%$ & \\
\hline BLT & $9.1 \%$ & $12.5 \%$ & \\
\hline Nephrectomy & $18 . \%$ & $0.0 \%$ & \\
\hline
\end{tabular}


Table 1 (continued)

\begin{tabular}{llll}
\hline Variable & 1st March-15th April 2019 & 1st March-15th April 2020 & $p$ value \\
\hline Antibiotic treatment & & & 0.542 \\
No & $0.0 \%$ & $12.5 \%$ & \\
Fluoroquinolones & $27.3 \%$ & $12.5 \%$ & \\
Cephalosporin & $36.4 \%$ & $25.0 \%$ & \\
Penicillins & $9.1 \%$ & $25.0 \%$ & \\
Carbapenems & $9.1 \%$ & $25.0 \%$ & \\
Others & $18.1 \%$ & $0.0 \%$ & 0.778 \\
Hospital stay (days) & $6.0 \pm 2.4(3.00-12.00)$ & $10.0 \pm 7.6(1.00-33.50)$ & \\
\hline
\end{tabular}

$p$ value chi-square test, $s C R$ serum creatinine, $E D$ emergency department, $W B C$ white blood cells, $U T I$ urinary tract infection, $U S$ ultrasonography; $C T$ computer tomography, ULT ureterolithotripsy, RIRS retrograde intrarenal surgery, $B L T$ bladder stone lithotripsy

\section{Compliance with Ethical Statement}

Conflict of interest The authors declare that they have no conflict of interest.

\section{References}

1. Livingston E, Bucher K (2020) Coronavirus disease 2019 (COVID-19) in Italy. JAMA. https://doi.org/10.1001/ jama.2020.4344 (Epub ahead of print)
2. Chan MC, Yeo SEK, Chong YL, Lee YM (2020) Stepping forwards: urologists' efforts during the COVID-19 outbreak in Singapore. Eur Urol. https://doi.org/10.1016/j.eururo.2020.03.004 (Epub ahead of print)

3. Stensland KD, Morgan TM, Moinzadeh A et al (2020) Considerations in the triage of urologic surgeries during the COVID-19 pandemic. Eur Urol 9:S0302-2838(20)30202-30205. https://doi. org/10.1016/j.eururo.2020.03.027

Publisher's Note Springer Nature remains neutral with regard to jurisdictional claims in published maps and institutional affiliations. 